\title{
Upregulation of catalase and downregulation of glutathione peroxidase activity in the kidney precede the development of hypertension in pre-hypertensive SHR
}

\author{
Arunkumar Sundaram $^{1}$, Lee Siew Keah ${ }^{1,2}$, Kuttulebbai Nainamohamed Salam Sirajudeen ${ }^{3}$ \\ and Harbindar Jeet Singh ${ }^{4}$
}

\begin{abstract}
Although oxidative stress has been implicated in the pathogenesis of hypertension in spontaneously hypertensive rats (SHRs), there is little information on the levels of primary antioxidant enzymes status (AOEs) in pre-hypertensive SHR. This study therefore determined the activities of primary AOEs and their mRNA levels, levels of hydrogen peroxide $\left(\mathrm{H}_{2} \mathrm{O}_{2}\right)$, malondialdehyde (MDA) and total antioxidant status (TAS) in whole kidneys of SHR and age-matched Wistar-Kyoto (WKY) rats aged between 2 and 16 weeks. Compared with age-matched WKY rats, catalase (CAT) activity was significantly higher from the age of 2 weeks $(P<0.001)$ and glutathione peroxide (GPx) activity was lower from the age of 3 weeks $(P<0.001)$ in SHR. CAT mRNA levels were significantly higher in SHR aged 2, 4, 6 and 12 weeks. GPx mRNA levels were significantly lower in SHR at 8 and 12 weeks. Superoxide dismutase activity or its mRNA levels were not different between the two strains. $\mathrm{H}_{2} \mathrm{O}_{2}$ levels were significantly lower in SHR from the age of 8 weeks $(P<0.01)$. TAS was significantly higher in SHR from the age of 3 weeks $(P<0.05)$. MDA levels were only significantly higher at 16 weeks of age in the SHR $(P<0.05)$. The data suggest that altered renal CAT and GPx mRNA expression and activity precede the development of hypertension in SHR. The raised CAT activity perhaps contributes to the higher TAS and lower $\mathrm{H}_{2} \mathrm{O}_{2}$ levels in SHR. In view of these findings, the precise role of oxidative stress in the pathogenesis of hypertension in SHR needs to be investigated further.
\end{abstract}

Hypertension Research (2013) 36, 213-218; doi:10.1038/hr.2012.163; published online 25 October 2012

Keywords: catalase; glutathione peroxidase; hydrogen peroxide; renal; SHR

\section{INTRODUCTION}

Renal inflammation associated with oxidative stress has been implicated in the pathogenesis of hypertension in spontaneously hypertensive rats (SHRs). ${ }^{1-5}$ Elevated levels of nitrotyrosine, 8-hydroxy-2-deoxyguanosine and $47^{\text {phox }}$ in the kidney of 3-week-old SHR indicate a disturbance in the redox system that precedes the onset of hypertension in SHR. ${ }^{1}$ Moreover, higher levels of renal nitrotyrosine, malondialdehyde (MDA), gp91 ${ }^{\text {phox }}, \mathrm{p} 22^{\text {phox }}$ in 24-week-old SHR imply a potential involvement of oxidative stress in the maintenance of hypertension. ${ }^{5}$ Besides, the reported increase in mean arterial pressure following direct infusion of hydrogen peroxide $\left(\mathrm{H}_{2} \mathrm{O}_{2}\right)$ into the renal medulla of normotensive rats suggests that the accumulated $\mathrm{H}_{2} \mathrm{O}_{2}$ in renal medulla might have a hypertensive consequence. ${ }^{6}$

Numerous studies have compared the activities of primary antioxidant enzymes (AOEs) in the kidneys of SHR with age-matched
Wistar-Kyoto (WKY) rats but with variable, and sometimes, conflicting results. Tang et al., ${ }^{7}$ for example, reported slightly lower glomerular copper/zinc-superoxide dismutase (CuZn-SOD), manganese-SOD (Mn-SOD), catalase (CAT) and glutathione peroxide (GPx) activities in adult SHR when compared with those in age-matched WKY rats. Similarly, Shou et al., ${ }^{8}$ found lower activities of CuZn-SOD, GPx and CAT in the renal medulla, lower levels of CuZn-SOD, GPx but higher activity of CAT in the renal cortex, whereas Mn-SOD activity was increased in both the cortex and medulla of stroke-prone SHR when compared with age-matched 32-week-old WKY rats. Zhan et al., ${ }^{9}$ on the other hand, found no differences in the activities of CuZn-SOD, CAT and GPx between SHR and age-matched 6-month-old WKY rats, but found some discordance in Mn-SOD, CuZn-SOD, CAT and GPx protein levels in the medulla and cortex of SHR. Lee et al., ${ }^{10}$ report significantly

${ }^{1}$ Department of Physiology, School of Medical Sciences, Universiti Sains Malaysia, Health Campus, Kubang Kerian, Kelantan, Malaysia; ${ }^{2}$ Faculty of Pharmaceutical Sciences, UCSI University, Jalan Menara Gading, UCSI Heights, Cheras, Kuala Lumpur, Malaysia; ${ }^{3}$ Department of Chemical Pathology, School of Medical Sciences, Universiti Sains Malaysia, Health Campus, Kubang Kerian, Kelantan, Malaysia and ${ }^{4}$ Faculty of Medicine, Universiti Teknologi MARA, Shah Alam, Selangor, Malaysia

Correspondence: Associate Professor Dr KNS Sirajudeen, Department of Chemical Pathology, School of Medical Sciences, Universiti Sains Malaysia, Health Campus, 16150 Kubang Kerian, Kelantan, Malaysia.

E-mail: sirajuden@kb.usm.my or knssiraj@yahoo.com

Received 4 July 2012; revised 7 August 2012; accepted 17 August 2012; published online 25 October 2012 
lower renal GPx activity from the age of 8 weeks onward in SHR when compared with age-matched WKY rats. Recently, Simao et al. ${ }^{11}$ reported renal cortical SOD-1 and SOD-3 protein levels that were lower at 3 months but not different at 12 months, and higher CAT protein levels at 3 months but not at 12 months when compared with age-matched WKY rats. They, however, found no differences in renal GPx at either 3 or 12 months between SHR and age-matched WKY rats.

Although the precise reason for the variable findings and their significance remains unclear, these studies nevertheless indicate the presence of an imbalance in the redox system in the kidney of SHR, which might have a role in the pathogenesis of hypertension. As most of these studies were performed in SHR with established hypertension, the differences in renal AOE activities, their mRNA expressions and protein levels prior and during the onset of hypertension has not been well documented. This information is useful in understanding the significance of oxidative stress in the pathogenesis of hypertension in this species. This study therefore determined the activities of primary AOEs including SOD, CAT and GPx in whole kidneys of SHR and WKY rats between the age of 2 and 16 weeks. To better understand the differences in the levels of renal AOE activity, mRNA levels of these enzymes including CuZn-SOD, Mn-SOD, CAT and GPx were also assessed. In addition, renal total antioxidant status (TAS), and levels of lipid peroxidation marker, MDA and $\mathrm{H}_{2} \mathrm{O}_{2}$ were also measured.

\section{METHODS}

\section{Animal experiment}

Male SHR and WKY rats, aged 2, 3, 4, 6, 8, 10, 12 and 16 weeks, were obtained from the Laboratory Animals Research Unit of Universiti Sains Malaysia, Health Campus, Kubang Kerian, Kelantan, Malaysia and housed at room temperature with a 12-12 h light-dark cycle (0600-1800 hours), and with access ad libitum to food and water throughout the experimental period. All animals were weaned at the age of 3 weeks and fed a locally manufactured standard rat chow (Goldcoin, Port Klang, Malaysia). The experimental design was approved by the Animal Ethics Committee of Universiti Sains Malaysia, Health Campus (PPSG/07(A)/084).

Arterial blood pressure was measured using a tail-cuff plethysmograph (Life Science IITC, Woodland Hills, CA, USA) at the age of 4, 6, 8, 10, 12 and 16 weeks. There were six rats in each age group. Following blood pressure measurements, body weight was recorded and the animals were lightly anesthetized with diethyl ether and decapitated using a small animal guillotine. No blood pressure measurements were performed on rats aged 2 and 3 weeks and they were decapitated immediately on collection from the animal unit. After decapitation, a laparotomy was performed and the left kidney was removed and cleaned with ice-cold normal saline. A portion of the kidney, weighing about $100 \mathrm{mg}$, was cut in cross-section and stored in RNA-later for gene expression analysis. The remaining part of the kidney was immediately frozen in liquid nitrogen. All the samples were then stored at $-80^{\circ} \mathrm{C}$ until processed.

\section{Homogenization and biochemical analyses}

Kidney homogenates $(10 \% \mathrm{w} / \mathrm{v})$ were prepared in ice-cold $\left(4^{\circ} \mathrm{C}\right)$ Tris- $\mathrm{HCl}$, pH 7.4 buffer in a homogenizer fitted with a Teflon pestle (Glas-Col, Vernon Hills, IL, USA) and homogenized at 800-1000 r.p.m. The homogenates were centrifuged (Eppendorf 5810R, Hamburg, Germany) at $1000 \times g$ at $4{ }^{\circ} \mathrm{C}$ for $10 \mathrm{~min}$. Supernatants were then stored at $-80^{\circ} \mathrm{C}$ until used for biochemical assays a few weeks later. Total protein in the homogenates was determined using Micro TP kit (Wako Pure Chemicals, Osaka, Japan). ${ }^{12}$ SOD activity was assayed according to the method of Misra and Fridovich. ${ }^{13}$ CAT activity was assayed according to the method of Goth. ${ }^{14}$ GPx activity was assayed according to the method of Flohe and Gunzler. ${ }^{15}$ TAS levels were measured according to the method of Koracevic et al. ${ }^{16}$ and MDA levels were assessed according to the method of Ohkawa et al. ${ }^{17} \mathrm{H}_{2} \mathrm{O}_{2}$ was estimated in the homogenate using a commercially available kit (Invitrogen, Carlsbad, CA, USA). The Amplex Red reagent (10-acetyl-3,7-dihydroxyphenoxazine) in combination with horseradish peroxidase has been used to detect $\mathrm{H}_{2} \mathrm{O}_{2}$ released from biological samples. In the presence of peroxidase, the Amplex Red reagent reacts with $\mathrm{H}_{2} \mathrm{O}_{2}$ in a $1: 1$ stoichiometry to produce the red-fluorescent oxidation product, resorufin, which can be detected at a wavelength of $560 \mathrm{~nm}$ spectrophotometrically.

\section{Real-time PCR analysis}

Total RNA from kidney tissue was extracted using RNeasy Mini Kit (Qiagen, Hilden, Germany). Two microgram of total RNA was synthesized into complementary DNA using Revert Aid H Minus First Strand cDNA Synthesis Kit (Fermentas, Hanover, MD, USA). The primer sequences for real-time PCR assay were taken from the literature: CuZn-SOD, GPx, $\beta$-actin, ${ }^{18} \mathrm{Mn}$-SOD ${ }^{19}$ and $\mathrm{CAT}^{20}$ Primers were purchased from Invitrogen. Real-time PCR analyses were performed using SYBR Green I PCR kit (Stratagene, La Jolla, CA, USA) via Mx 3000P instrument (Stratagene). The conditions of real-time PCR were as follows: (1) initial PCR activation $-95^{\circ} \mathrm{C}, 10 \mathrm{~min}$, (2) 3-step cycling for 35 cycles involving (a) denaturation $-95^{\circ} \mathrm{C}, 30 \mathrm{~s}$, (b) annealing $-58^{\circ} \mathrm{C}, 1 \mathrm{~min}$ and (c) extension $-72{ }^{\circ} \mathrm{C}, 1 \mathrm{~min}$, followed by a dissociation phase. The PCR products were also subsequently subjected to $1.5 \%$ agarose gel electrophoresis for the confirmation of PCR amplicons. Serial dilution of complementary DNA was performed for each set of genes and primers to validate the amplification efficiencies of real-time PCR analysis and the efficiencies were around $90-100 \%$. Relative expression of mRNA was then determined by the comparative $2^{-\Delta \Delta C}$ T method. ${ }^{21}$

\section{Statistical analysis}

Data were analyzed using one-way analysis of variance and expressed as mean \pm s.e.m. A value of ' $P$ ' of $<0.05$ was considered statistically significant.

\section{RESULTS}

\section{Body weight and systolic blood pressure}

Body weight was significantly lower in SHR at all age groups when compared with age-matched WKY rats $(P<0.001$; Table 1$)$. Systolic blood pressure was significantly higher in SHR from the age of 6 weeks $(P<0.001$; Table 1$)$.

Table 1 Body weight and systolic blood pressure in SHR and WKY rats

\begin{tabular}{|c|c|c|c|c|c|c|c|c|}
\hline Age & $2 W k$ & $3 W k$ & $4 W k$ & $6 W k$ & $8 W k$ & $10 W k$ & $12 W k$ & $16 W k$ \\
\hline \multicolumn{9}{|c|}{ Body weight (g) } \\
\hline WKY & $28 \pm 1.1$ & $48 \pm 1.2$ & $58 \pm 2.3$ & $160 \pm 3.3$ & $198 \pm 2.4$ & $245 \pm 3.8$ & $268 \pm 5.7$ & $347 \pm 7.6$ \\
\hline SHR & $19 \pm 1.1^{* * *}$ & $39 \pm 0.7 * * *$ & $46 \pm 2.3^{* *}$ & $146 \pm 4.2^{* * *}$ & $162 \pm 6.1^{* * *}$ & $179 \pm 4.5^{* * *}$ & $212 \pm 7.3^{* * *}$ & $256 \pm 3.8^{* * *}$ \\
\hline \multicolumn{9}{|c|}{ Systolic blood pressure $(\mathrm{mm} \mathrm{Hg})$} \\
\hline WKY & - & - & $116 \pm 2.1$ & $121 \pm 2.3$ & $123 \pm 2.5$ & $124 \pm 3.6$ & $122 \pm 3.2$ & $129 \pm 2.9$ \\
\hline SHR & - & - & $118 \pm 3.2$ & $148 \pm 2.8^{* * *}$ & $151 \pm 3.6^{* * *}$ & $170 \pm 4.8^{* * *}$ & $168 \pm 3.6^{* * *}$ & $170 \pm 3.2^{* * *}$ \\
\hline
\end{tabular}

Abbreviations: SHR, spontaneously hypertensive rats; Wk, weeks; WKY, Wistar-Kyoto.

${ }^{* *} P<0.01,{ }^{* * *} P<0.001$ when compared with age-matched WKY rats. 


\section{Renal AOE status}

There was no significant difference in total SOD activity (Figure 1), and mRNA levels of CuZn-SOD or Mn-SOD (Table 2) between SHR and age-matched WKY. CAT activity was significantly higher in SHR from the age of 2 weeks $(P<0.001$; Figure 2$)$. CAT mRNA levels were higher in all the measured weeks and statistical significance was evident between $2(P<0.05), 4 \quad(P<0.05), 6(P<0.05)$ and 12 $(P<0.05)$ weeks old SHR and age-matched WKY rats (Table 2$).$ GPx activity was significantly lower from the age of 3 weeks $(P<0.001$; Figure 3$)$, and although GPx mRNA levels were consistently lower in SHR than those in WKY rats, significant difference was, however, only evident at $8(P<0.05)$ and $10(P<0.01)$ weeks of age (Table 2).

\section{Renal TAS and MDA levels}

TAS was consistently higher in SHR than that in age-matched WKY rats, reaching statistical significance at $3(P<0.05), 4(P<0.05), 10$ $(P<0.05), 12(P<0.05)$ and $16(P<0.01)$ weeks of age (Table 3$)$. However, MDA levels were only significantly higher in SHR at 16 weeks of age $(P<0.05$; Table 3$)$.

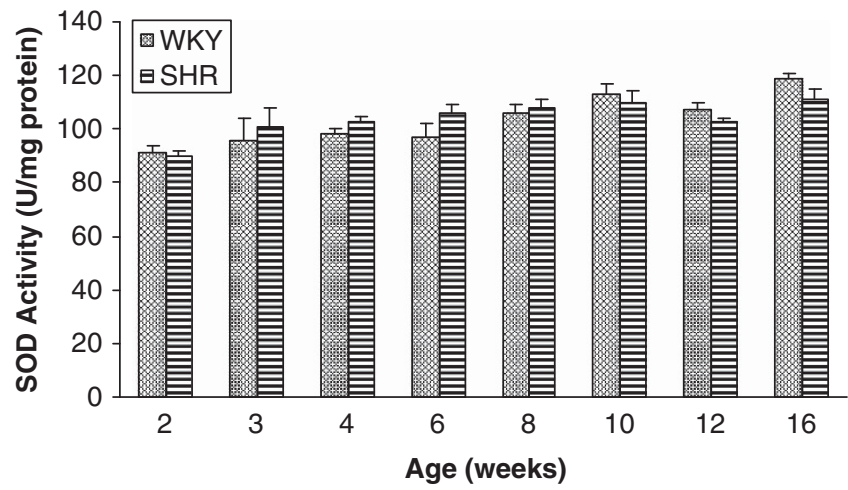

Figure 1 Renal superoxide dismutase (SOD) activities in spontaneously hypertensive rat (SHR) and Wistar-Kyoto (WKY) rats. $(U=$ the amount of SOD that inhibits the rate of auto-oxidation of epinephrine by 50\%).

\section{Renal $\mathrm{H}_{2} \mathrm{O}_{2}$ levels}

Levels of $\mathrm{H}_{2} \mathrm{O}_{2}$ were consistently lower in SHR from the age of 2 weeks, but statistical significance was only evident from the age of 8 weeks onward $(P<0.01$; Table 4$)$.

\section{DISCUSSION}

The main findings of this study include: (i) higher CAT activity and its relative mRNA levels in the kidneys of SHR from the age of 2 weeks (Figure 2 and Table 2), (ii) lower kidney GPx activity in SHR from the age of 3 weeks and lower relative GPx mRNA levels at 8 and 10 weeks of age (Figure 3 and Table 2), (iii) higher renal TAS in SHR from the age of 3 weeks and onward (Table 3), (iv) higher renal MDA levels at 16 weeks (Table 3), (v) lower levels of renal $\mathrm{H}_{2} \mathrm{O}_{2}$ in SHR from the age of 8 weeks (Table 4) and (vi) no significant difference in renal total SOD activity or the relative SOD mRNA levels of CuZn-SOD and Mn-SOD (Figure 1 and Table 2) when compared with age-matched WKY rats.

Although a significantly elevated renal CAT activity in 6- and 16 -week old, ${ }^{22}$ and older $\mathrm{SHR}^{8,23}$ has been reported before, this is the first time that altered renal CAT expression and activity has been reported in pre-hypertensive SHR. The demonstration of higher CAT

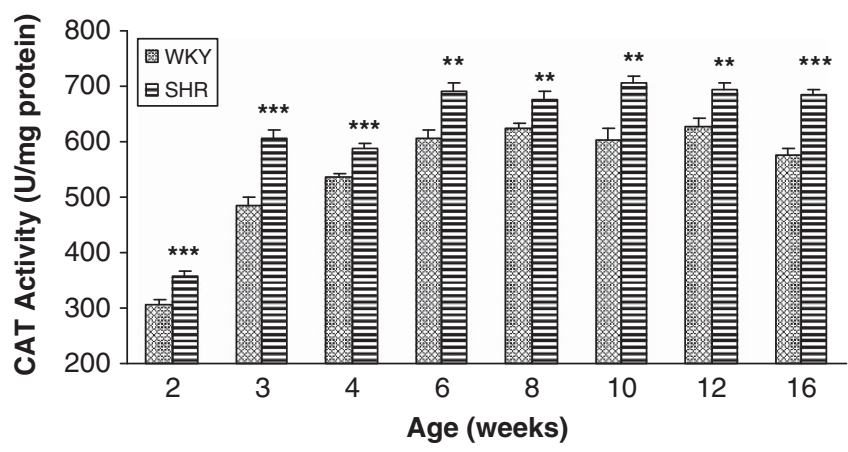

Figure 2 Renal catalase (CAT) activities in spontaneously hypertensive rat (SHR) and age-matched Wistar-Kyoto (WKY) rats. ${ }^{*} P<0.01,{ }^{*} * * P<0.001$ when compared to age-matched WKY rats, $(U=\mu \mathrm{mol}$ of hydrogen peroxide decomposed per minute).

Table 2 mRNA levels of CuZn-SOD, Mn-SOD, CAT and GPx in the kidneys of SHR and age-matched WKY rats

\begin{tabular}{|c|c|c|c|c|c|c|c|}
\hline Age & $2 W k$ & $4 W k$ & $6 W k$ & $8 W k$ & $10 W k$ & $12 W k$ & $16 W k$ \\
\hline \multicolumn{8}{|c|}{ CUZn-SOD mRNA } \\
\hline WKY & - & $1.00 \pm 0.12$ & $1.00 \pm 0.16$ & $1.00 \pm 0.08$ & $1.00 \pm 0.23$ & $1.00 \pm 0.08$ & $1.00 \pm 0.15$ \\
\hline SHR & - & $0.94 \pm 0.11$ & $0.9 \pm 0.13$ & $1.15 \pm 0.06$ & $0.87 \pm 0.15$ & $1.07 \pm 0.07$ & $0.97 \pm 0.17$ \\
\hline \multicolumn{8}{|c|}{$M n-S O D$ mRNA } \\
\hline WKY & - & $1.00 \pm 0.09$ & $1.00 \pm 0.10$ & $1.00 \pm 0.15$ & $1.00 \pm 0.23$ & $1.00 \pm 0.22$ & $1.00 \pm 0.17$ \\
\hline SHR & - & $1.11 \pm 0.03$ & $0.84 \pm 0.19$ & $0.99 \pm 0.09$ & $0.85 \pm 0.15$ & $1.06 \pm 0.14$ & $0.93 \pm 0.05$ \\
\hline \multicolumn{8}{|c|}{ CAT mRNA } \\
\hline WKY & $1.00 \pm 0.28$ & $1.00 \pm 0.07$ & $1.00 \pm 0.16$ & $1.00 \pm 0.15$ & $1.00 \pm 0.13$ & $1.00 \pm 0.11$ & $1.00 \pm 0.16$ \\
\hline SHR & $1.82 \pm 0.21^{*}$ & $1.35 \pm 0.13^{*}$ & $1.40 \pm 0.04^{*}$ & $1.23 \pm 0.05$ & $1.13 \pm 0.12$ & $1.23 \pm 0.03^{*}$ & $1.09 \pm 0.16$ \\
\hline \multicolumn{8}{|c|}{$G P x m R N A$} \\
\hline WKY & $1.00 \pm 0.15$ & $1.00 \pm 0.07$ & $1.00 \pm 0.11$ & $1.00 \pm 0.16$ & $1.00 \pm 0.16$ & $1.00 \pm 0.12$ & $1.00 \pm 0.09$ \\
\hline SHR & $1.00 \pm 0.14$ & $0.97 \pm 0.11$ & $0.81 \pm 0.11$ & $0.71 \pm 0.15^{*}$ & $0.61 \pm 0.02 * *$ & $1.27 \pm 0.12$ & $0.79 \pm 0.17$ \\
\hline
\end{tabular}


activity and its mRNA levels from as early as 2 weeks in SHR suggests that CAT expression and activity might be one of the primary abnormalities in SHR that might have a role in the pathogenesis of hypertension. CAT is known to have a significant role in the development of tolerance to oxidative stress in cells, ${ }^{24,25}$ particularly when there is decreased GPx activity ${ }^{18}$ or elevated $\mathrm{H}_{2} \mathrm{O}_{2}$ levels. ${ }^{26,27}$ GPx activity was slightly but not significantly lower in SHR at 2 weeks of age when compared with WKY rats (Figure 3) and whether the slightly lower GPx activity at 2 weeks of age and afterward was due to the overexpression of CAT or vice versa in this study is uncertain.

It has recently been proposed that moderate changes in GPx-1 activity could result in major effects on vascular function, and that a deficiency of this enzyme might be one of those as-yet-undefined enzymes that contribute to the development of hypertension. ${ }^{28}$ Interestingly, significantly lower renal GPx activity from as early as

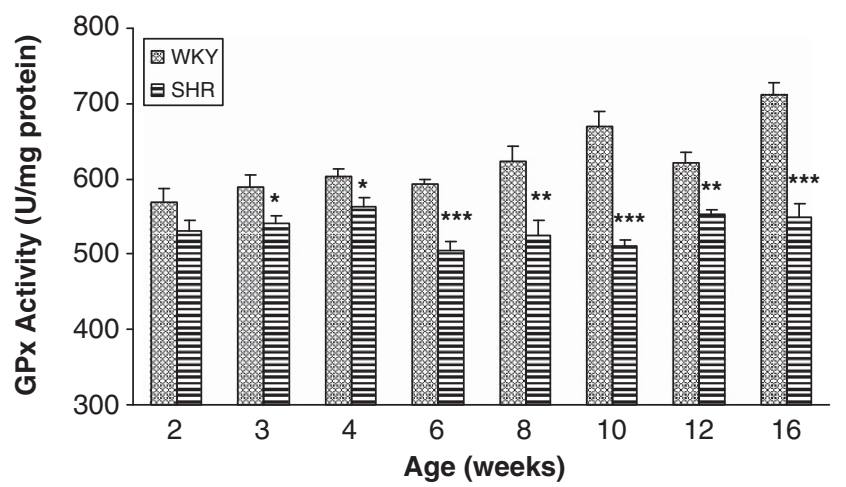

Figure 3 Renal glutathione peroxide (GPx) activities between spontaneously hypertensive rat (SHR) and age-matched Wistar-Kyoto (WKY) rats. ${ }^{*} P<0.05,{ }^{* *} P<0.01,{ }^{* * *} P<0.001$ when compared with age-matched WKY rats, $(U=\mathrm{nmol}$ of $\mathrm{NADPH}$ oxidized per minute).
3 weeks was evident in this study, and it preceded the development of hypertension (Figure 3). The lower GPx activity in SHR also coincided with lower mRNA levels in some age groups, particularly in groups aged 8 and 10 weeks (Table 2). The finding of lower GPx activity at 3 weeks of age is a little different from our previous report where significantly lower GPx activity was only evident from the age of 8 weeks. ${ }^{10}$ The exact reason for this difference is not clear as similar method for its estimation was used in both studies. Notwithstanding this difference, the overall finding of lower GPx activity at 3 weeks of age might imply an over compensation by the elevated CAT activity causing a decrease in the substrate and therefore in GPx activity and its subsequent mRNA expression and perhaps also the higher TAS and unaltered levels of MDA between 2 and 12 weeks. Estimation of markers of protein and DNA oxidation might provide a clearer picture on this.

Although the precise significance of the differences in the pattern of CAT and GPx activity and their respective mRNA expressions in SHR and WKY rats in this study remains unclear, it is nevertheless interesting to note that the differences in renal mRNA expression and activities of CAT and, to some extent, that of GPx, between SHR and WKY rats reported in this study, became apparent before the actual rise in blood pressure in SHR. Whether similar changes in these enzymes are also evident in other organs is not certain as they were not determined in this study but the presence of these differences in the kidney during the pre-hypertensive period suggests a potential role for them in the pathogenesis of hypertension. Their measurement in other tissues like the heart and blood vessels would further indicate their involvement in the development of hypertension in SHR. In addition, the use of CAT inhibitor during the pre-hypertensive stage might further elucidate the role of elevated CAT in the pathogenesis of hypertension in this species, although melatonin supplementation during the antenatal and postnatal period was found to significantly increase CAT expression and activity and delay the rise in blood pressure in hypertensive rats. ${ }^{22}$

Table 3 Renal TAS and MDA levels in SHR and age-matched WKY rats

\begin{tabular}{|c|c|c|c|c|c|c|c|c|}
\hline Age & $2 W k$ & $3 W k$ & $4 W k$ & $6 W k$ & $8 W k$ & $10 W k$ & $12 W k$ & 16 Wk \\
\hline \multicolumn{9}{|c|}{ TAS ( $\mu \mathrm{mol}$ of uric acid equivalent per mg protein) } \\
\hline WKY & $3.46 \pm 0.06$ & $4.04 \pm 0.06$ & $4.20 \pm 0.08$ & $5.22 \pm 0.12$ & $5.49 \pm 0.13$ & $5.04 \pm 0.12$ & $4.99 \pm 0.17$ & $4.71 \pm 0.12$ \\
\hline SHR & $3.63 \pm 0.08$ & $4.39 \pm 0.11^{*}$ & $4.49 \pm 0.11^{*}$ & $5.59 \pm 0.16$ & $5.69 \pm 0.10$ & $5.54 \pm 0.16^{*}$ & $5.47 \pm 0.12 *$ & $5.33 \pm 0.11^{* *}$ \\
\hline \multicolumn{9}{|c|}{ MDA (nmol per mg protein) } \\
\hline WKY & $2.43 \pm 0.10$ & $2.51 \pm 0.10$ & $2.52 \pm 0.10$ & $2.38 \pm 0.07$ & $2.40 \pm 0.07$ & $2.55 \pm 0.07$ & $2.49 \pm 0.13$ & $2.56 \pm 0.08$ \\
\hline SHR & $2.74 \pm 0.19$ & $2.60 \pm 0.11$ & $2.65 \pm 0.12$ & $2.40 \pm 0.07$ & $2.37 \pm 0.05$ & $2.46 \pm 0.11$ & $2.50 \pm 0.10$ & $2.91 \pm 0.11^{*}$ \\
\hline
\end{tabular}

Abbreviations: MDA, malondialdehyde; SHR, spontaneously hypertensive rats; TAS, total antioxidant status; Wk, weeks; WKY, Wistar-Kyoto.

${ }^{*} P<0.05,{ }^{* *} P<0.01$ when compared with age-matched WKY rats.

Table 4 Renal $\mathrm{H}_{2} \mathrm{O}_{2}$ levels in SHR and age-matched WKY rats

\begin{tabular}{|c|c|c|c|c|c|c|}
\hline & $2 W k$ & $4 W k$ & $6 W k$ & $8 W k$ & $12 W k$ & $16 W k$ \\
\hline \multicolumn{7}{|c|}{$\mathrm{H}_{2} \mathrm{O}_{2}\left(\mu \mathrm{MI}^{-1}\right)$} \\
\hline WKY & $34.36 \pm 1.17$ & $46.61 \pm 1.63$ & $55.89 \pm 3.48$ & $63.27 \pm 4.33$ & $66.25 \pm 0.82$ & $62.46 \pm 4.41$ \\
\hline SHR & $31.53 \pm 1.32$ & $45.48 \pm 0.87$ & $49.92 \pm 2.41$ & $45.36 \pm 2.48^{* *}$ & $51.97 \pm 1.26^{* * *}$ & $40.51 \pm 3.44^{* *}$ \\
\hline
\end{tabular}

Abbreviations: $\mathrm{H}_{2} \mathrm{O}_{2}$, hydrogen peroxide; SHR, spontaneously hypertensive rats; Wk, weeks; WKY, Wistar-Kyoto.

${ }^{* *} P<0.01,{ }^{* * *} P<0.001$ compared with age-matched WKY. 
CAT mRNA level and its activity are usually upregulated when there is a disturbance in the oxidative status, particularly in $\mathrm{H}_{2} \mathrm{O}_{2}$ levels. ${ }^{26,27}$ Surprisingly, renal $\mathrm{H}_{2} \mathrm{O}_{2}$ levels were consistently lower in SHR, particularly from 8 weeks onward (Table 4) and therefore, the raised levels of CAT may not be due to elevated $\mathrm{H}_{2} \mathrm{O}_{2}$. The lower levels of $\mathrm{H}_{2} \mathrm{O}_{2}$ could, on the other hand, be due to the higher CAT activity. The finding of lower levels of $\mathrm{H}_{2} \mathrm{O}_{2}$ in this study is in contrast to earlier reports where elevated levels of $\mathrm{H}_{2} \mathrm{O}_{2}$ production were reported in immortalized renal proximal tubular epithelial cells from 4- to 8-week-old SHR, and renal cortical cells from 3-month but not 12-month-old SHR. ${ }^{29}$ The reason for this difference is not apparent apart from the fact that $\mathrm{H}_{2} \mathrm{O}_{2}$ levels were measured in young SHR and in whole kidney homogenates in this study, whereas in the above studies measurements were made in cells in vitro. The finding of lower $\mathrm{H}_{2} \mathrm{O}_{2}$ levels in this study is also in conflict with its proposed role in the pathogenesis of hypertension. In a recent study, for example, infusion of high doses of $\mathrm{H}_{2} \mathrm{O}_{2}\left(4.0 \mu \mathrm{mol} \mathrm{kg}{ }^{-1}\right.$ per day $)$ for a period of 7 days, while increasing NOS expression in the kidneys, caused a significant increase in systolic blood pressure in normotensive WKY rats. ${ }^{30}$ Furthermore, these researchers also report that infusion of $\mathrm{H}_{2} \mathrm{O}_{2}$ did not alter renal reduced nicotinamide adenine dinucleotide phosphate oxidase activity. Incidentally, reduced nicotinamide adenine dinucleotide phosphate oxidase is considered to be the major source of superoxide generation. ${ }^{31}$ The lower $\mathrm{H}_{2} \mathrm{O}_{2}$ levels in SHR in this study seems to suggest that $\mathrm{H}_{2} \mathrm{O}_{2}$ might not have a significant direct role in the pathogenesis of hypertension in this strain of rat as despite the lower $\mathrm{H}_{2} \mathrm{O}_{2}$ levels, systolic blood pressure still increased in the SHR.

To examine if the differences in the pattern of CAT and GPx activity between the two strains had any impact on the antioxidant capabilities of the renal tissue, TAS was measured in these tissues. TAS was significantly higher in SHR from the age of 3 weeks when compared with age-matched WKY rats (Table 3). To our knowledge, this is the first study that has measured the overall TAS in the kidneys of SHR and WKY rats between the age of 2 and 16 weeks. Higher TAS has been reported in 16-week-old SHR recently by us. ${ }^{22}$ Lower renal TAS has also been previously reported in 22-week-old SHR. ${ }^{32}$ It is possible that the higher TAS in this study might be due to the higher CAT activity as CAT has one of the highest turnover rates of all enzymes where one molecule of CAT can convert millions of molecules of $\mathrm{H}_{2} \mathrm{O}_{2}$ to water and oxygen per second. ${ }^{33,34}$

Given the higher TAS in the renal tissue of SHR, we then compared the levels of MDA, a marker of lipid peroxidation, between the two strains of rat over the study period. MDA levels were not different between the two strains until 12 weeks of age, but were significantly higher at the age of 16 weeks in SHR (Table 4), indicating the appearance of oxidative stress. The lack of significant difference in MDA till the age of 12 weeks might be because of the higher TAS, limiting lipid peroxidation until about the age of 12 weeks, after which it might not have been able to do so.

SOD is the primary enzyme that is involved in dismutasing superoxide. Inhibition of SOD activity by continuous infusion of diethyldithiocarbamic acid (SOD inhibitor) into the renal medulla has been shown to increase blood pressure in normotensive rats. ${ }^{6}$ In addition, subunits of renal nicotinamide adenine dinucleotide phosphate oxidase (superoxide generator) or its activity have also been reported to be augmented in the pre-hypertensive ${ }^{1}$ and hypertensive SHR. ${ }^{5,9,30}$ Our results showed no significant difference in total SOD activity (Figure 1) or in the mRNA expressions of CuZnSOD and Mn-SOD (Table 2) between SHR and WKY rats aged between 2 and 16 weeks. This has also previously been reported by
Lee et al. ${ }^{22}$ Numerous other studies too have reported similar findings of unchanged SOD activity or its protein levels in the kidneys of adult SHR. ${ }^{23,30,35}$ Renal morphology or urine protein excretion was not examined in this study. However, our earlier observations in the same species (unpublished data) did not reveal any significant differences in renal morphology or urinary protein excretion between SHR and WKY rats aged 4-16 weeks, except after 48 weeks of age when significant decreases in glomerular filtration rate and glomerular necrosis was evident in SHR.

In conclusion, the data suggest altered CAT and GPx mRNA expression and activity patterns in SHR occur very early during the pre-hypertensive period in SHR and might have an important role in the pathogenesis of high blood pressure in this strain of rat. The raised CAT activity perhaps also contributed to the higher TAS and lower $\mathrm{H}_{2} \mathrm{O}_{2}$ levels in SHR. In view of the findings, the precise role of oxidative stress in the pathogenesis of hypertension in SHR needs to be investigated further, in particular the significance of the altered CAT and GPx activities.

\section{ACKNOWLEDGEMENTS}

This study was supported by the Fundamental Research Grant Scheme (FRGS/ 203/PPSP/6170021) provided by Ministry of Higher Education Malaysia and the Research University (RU 1001/PPSP/811018) provided by Universiti Sains Malaysia. AS is sponsored by Ministry of Science Technology and Innovation Malaysia. LSK is given Fellowship from University Sains Malaysia.

1 Biswas SK, De Faria JB. Which comes first: renal inflammation or oxidative stress in spontaneously hypertensive rats? Free Radic Res 2007; 41: 216-224.

2 Rodriguez-Iturbe B, Vaziri ND, Herrera-Acosta J, Johnson RJ. Oxidative stress, renal infiltration of immune cells, and salt-sensitive hypertension: all for one and one for all. Am J Physiol Renal Physiol 2004; 286: F606-F616.

3 Vaziri ND. Causal link between oxidative stress, inflammation, and hypertension. Iran J Kidney Dis 2008; 2: 1-10.

4 Vaziri ND, Rodriguez-Iturbe B. Mechanisms of disease: oxidative stress and inflammation in the pathogenesis of hypertension. Nat Clin Pract Nephrol 2006; 2: 582-593.

5 Zhan CD, Sindhu RK, Vaziri ND. Up-regulation of kidney $\mathrm{NAD}(\mathrm{P}) \mathrm{H}$ oxidase and calcineurin in SHR: reversal by lifelong antioxidant supplementation. Kidney Int 2004; 65: 219-227.

6 Makino A, Skeleton MM, Zou A, Cowley AW. Increased renal Medullary H2O2 leads to hypertension. Hypertension 2003; 42: 25-30.

7 Tang Z, Shou I, Wang LN, Fukui M, Tomino Y. Effects of antihypertensive drugs or glycemic control on antioxidant enzyme activities in spontaneously hypertensive rats with diabetes. Nephron 1997; 76: 323-330.

8 Shou I, Wang LN, Suzuki S, Fukui M, Tomino Y. Effects of antihypertensive drugs on antioxidant enzyme activities and renal function in stroke-prone spontaneously hypertensive rats. Am J Med Sci 1997; 314: 377-384.

9 Zhan CD, Sindhu RK, Pang J, Ehdaie A, Vaziri ND. Superoxide dismutase, catalase and glutathione peroxidase in the spontaneously hypertensive rat kidney: effect of antioxidant-rich diet. J Hypertens 2004; 22: 2025-2033.

10 Lee SK, Arunkumar S, Sirajudeen KN, Singh HJ. Glutathione system in young spontaneously hypertensive rats. J Physiol Biochem 2010; 66: 321-327.

11 Simao S, Gomes P, Pinto V, Silva E, Amaral JS, Igreja B, Afonso J, Serrao MP, Pinho MJ, Soares-Da-Silva P. Age-related changes in renal expression of oxidant and antioxidant enzymes and oxidative stress markers in male SHR and WKY rats. Exp Gerontol 2011; 46: 468-474.

12 Watanabe N, Kamei S, Ohkubo A, Yamanaka M, Ohsawa S, Makino K, Tokuda K. Urinary protein as measured with a pyrogallol red-molybdate complex, manually and in a Hitachi 726 automated analyzer. Clin Chem 1986; 32: 1551-1554.

13 Misra HP, Fridovich I. The role of superoxide anion in the autoxidation of epinephrine and a simple assay for superoxide dismutase. J Biol Chem 1972; 247: 3170-3175.

14 Goth L. A simple method for determination of serum catalase activity and revision of reference range. Clin Chim Acta 1991; 196: 143-151.

15 Flohe L, Gunzler WA. Assay of glutathione peroxidase. In: Colowick SP and Kaplan NO (Eds) Methods in Enzymology. 105, Academic Press, Orlando, FL, 1984, pp 114-121.

16 Koracevic D, Koracevic G, Djordjevic V, Andrejevic S, Cosic V. Method for the measurement of antioxidant activity in human fluids. J Clin Path 2001; 54: 356-361.

17 Ohkawa H, Ohishi N. Yagi K. Assay for lipid peroxides in animal tissues by thiobarbituric acid reaction. Anal Biochem 1979; 95: 351-358. 
18 Bhor VM, Raghuram N, Sivakami S. Oxidative damage and altered antioxidant enzyme activities in the small intestine of streptozotocin-induced diabetic rats. Int J Biochem Cell Biol 2004; 36: 89-97.

19 Cederberg J, Galli J, Luthman H, Eriksson UJ. Increased mRNA levels of Mn-SOD and catalase in embryos of diabetic rats from a malformation-resistant strain. Diabetes 2000; 49: 101-107.

20 Tam NN, Gao Y, Leung YK, Ho SM. Androgenic regulation of oxidative stress in the rat prostate: involvement of $\mathrm{NAD}(\mathrm{P}) \mathrm{H}$ oxidases and antioxidant defense machinery during prostatic involution and regrowth. Am J Pathol 2003; 163: 2513-2522.

21 Livak KJ, Schmittgen TD. Analysis of relative gene expression data using real-time quantitative PCR and the 2(-delta delta C(T)) method. Methods 2001; 25: 402-408.

22 Lee SK, Sirajudeen KN, Sundaram A, Zakaria R, Singh HJ. Effects of antenatal, postpartum and post-weaning melatonin supplementation on blood pressure and renal antioxidant enzyme activities in spontaneously hypertensive rats. J Physiol Biochem 2011; 67: 249-257.

23 Hong $\mathrm{H}$, Johnson P. Antioxidant enzyme activities and lipid peroxidation levels in exercised and hypertensive rat tissues. Int J Biochem Cell Biol 1995; 27: 923-931.

24 Powers SK, Sen CK. Physiological antioxidants and exercise training. In: Sen CK Packer L and Hänninen OOP (eds). Handbook of Oxidants and Antioxidants in Exercise. Elsevier Science B.V., Amsterdam, The Netherlands, 2000, pp 221-242.

25 Wassmann S, Wassmann K, Nickenig G. Modulation of oxidant and antioxidant enzyme expression and function in vascular cells. Hypertension 2004; 44: 381-386.

26 Yamada M, Hashinaka K, Inazawa J, Abe T. Expression of catalase and myeloperoxidase genes in hydrogen peroxide-resistant HL-60 cells. DNA Cell Biol 1991; 10: 735-742.
27 Spitz DR, Adams DT, Sherman CM, Roberts RJ. Mechanisms of cellular resistance to hydrogen peroxide, hyperoxia, and 4-hydroxy-2-nonenal toxicity: the significance of increased catalase activity in H2O2-resistant fibroblasts. Arch Biochem Biophys 1992; 292: 221-227.

28 Brosnan MJ. One step beyond: glutathione peroxidase and endothelial dysfunction. Hypertension 2008; 51: 825-826.

29 Pedrosa R, Villar VA, Pascua AM, Simao S, Hopfer U, Jose PA, Soares-Da-Silva P. $\mathrm{H}_{2} \mathrm{O}_{2}$ stimulation of the $\mathrm{Cl}-/ \mathrm{HCO} 3-$ exchanger by angiotensin $\mathrm{II}$ and angiotensin II type 1 receptor distribution in membrane microdomains. Hypertension 2008; 51: 1332-1338.

30 Cao P, Ito O, Guo Q, Ito D, Muroya Y, Rong R, Mori T, Ito S, Kohzuki M. Endogenous hydrogen peroxide up-regulates the expression of nitric oxide synthase in the kidney of SHR. J Hypertens 2011; 29: 1167-1174.

31 Pechanova O, Jendekova L, Vrankova S. Effect of chronic apocynin treatment on nitric oxide and reactive oxygen species production in borderline and spontaneous hypertension. Pharmacol Rep 2009; 61: 116-122.

32 Sun L, Gao YH, Tian DK, Zheng JP, Zhu CY, Ke Y, Bian K. Inflammation of different tissues in spontaneously hypertensive rats. Acta Physiologica Sinica 2006; 58: 318-323.

33 Kirkman HN, Galiano S, Gaetani GF. The function of catalase-bound NADPH. J Bio Chem 1987; 262: 660-666.

34 Kirkman HN, Gaetani GF. Mammalian catalase: a venerable enzyme with new mysteries. Trends Biochem Sci 2007; 32: 44-50.

35 Fortepiani LA, Reckelhoff JF. Increasing oxidative stress with molsidomine increases blood pressure in genetically hypertensive rats but not normotensive controls. Am J Physiol Regul Integr Comp Physiol 2005; 289: R763-R770. 